\title{
Distortion and destruction of colloidal flocks in disordered environments
}

\author{
Alexandre Morin, Nicolas Desreumaux, Jean-Baptiste Caussin and Denis Bartolo*
}

\begin{abstract}
How do flocks, herds and swarms move through disordered environments? The answer to this question is crucial not only to animal groups in the wild, but also to effectively all applications of collective robotics and active materials composed of synthetic motile units ${ }^{1-14}$. In stark contrast, aside from rare exceptions ${ }^{15-17}$, our physical understanding of flocking has so far been limited to homogeneous media ${ }^{18-20}$. Here we explain how collective motion survives in geometrical disorder. To do so, we combine experiments and analytical theory to examine motile colloids cruising between randomly positioned microfabricated obstacles. We elucidate how disorder and bending elasticity compete to channel the flow of polar flocks along sparse river networks akin those found beyond plastic depinning in driven condensed matter ${ }^{21}$. Further increasing the disorder, we demonstrate that collective motion is suppressed in the form of a first-order phase transition generic to all polar active materials.
\end{abstract}

We use the experimental system introduced in refs 10,22, which consists of colloidal rollers powered by the so-called Quincke electro-rotation mechanism ${ }^{23}$ (see Methods and Supplementary Methods). The motile colloids experience both hydrodynamic and electrostatic interactions which promote alignment of their translational velocity ${ }^{10,22}$. When the roller packing fraction, $\rho$, exceeds $3 \times 10^{-3}$, these polar interactions overcome rotational diffusion and macroscopic collective motion emerges ${ }^{10,22}$. In the homogeneous slab geometry shown in Fig. 1a, a seven-millimetrelong flock spontaneously forms and cruises through a dilute ensemble of rollers moving isotropically (see Supplementary Movie 1). The flock has a sharp front, a long tail, and endlessly cruises at a constant speed along the $x$-axis, bouncing back and forth on the confining walls. The flock speed $c_{\mathrm{F}}$ is found to be equal to the speed of an isolated roller $v_{0}=1.4 \pm 0.1 \mathrm{~mm} \mathrm{~s}^{-1}$.

Can flocks propagate in disorder media? How does this brokensymmetry phase survive to geometrical disorder? To answer these questions, we include randomly distributed circular obstacles of radius $a=5 \mu \mathrm{m}$ in the microfluidic channel. When the obstacle packing fraction $\phi_{0}$ is small, collective motion still emerges according to the same nucleation and propagation scenario (see Fig. $1 \mathrm{~b}$ and Supplementary Movie 2). However, as $\phi_{\mathrm{o}}$ exceeds a critical value, $\phi_{\mathrm{o}}^{\star}$, the obstacle collisions suppress any form of global orientational order and macroscopic transport. Correlated motion persists only at short scales, as illustrated in Supplementary Movie 3. As expected, dense flocks are more robust to disorder and $\phi_{0}^{\star}$ increases monotonically with the roller fraction $\rho$ (Fig. 1c).

In all that follows, the sole control parameter of our experiments is the obstacle fraction $\phi_{0}$. The roller fraction is set to a constant value above the flocking threshold in a obstacle-free channel, $\rho=(1.02 \pm 0.06) \times 10^{-2}$. A natural order parameter for the flocking transition is the magnitude $J_{x}$ of the roller current

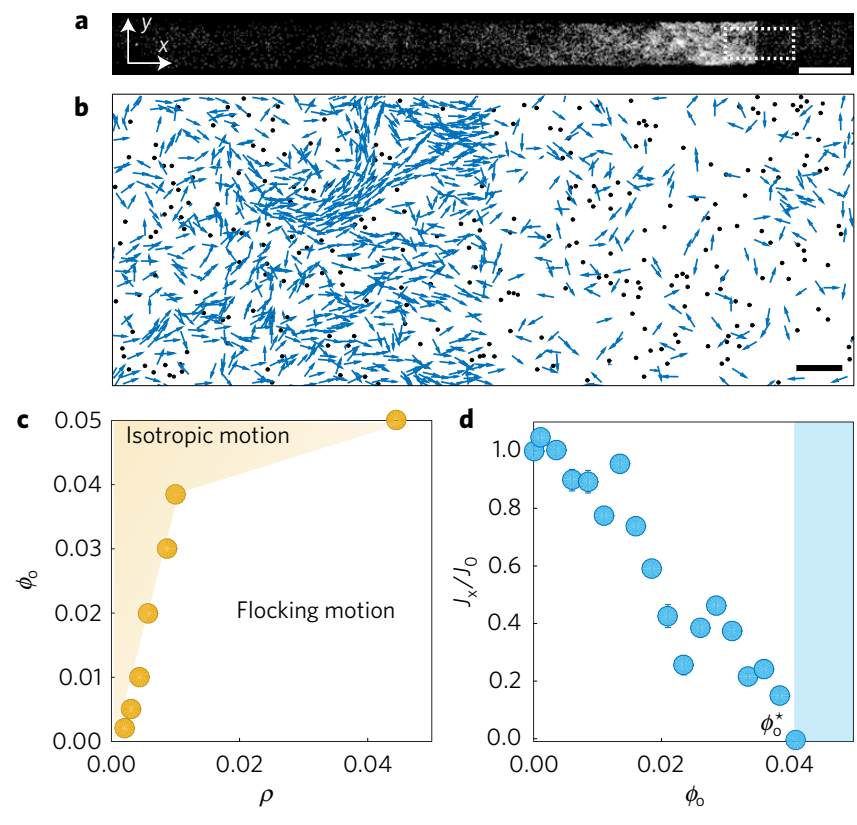

Figure 1 | Emergence and suppression of collective motion. a, Stitched fluorescent images of a 7-mm-long colloidal flock cruising in a rectangular channel. Total number of colloids: 8,500. Dotted rectangle: region in which the velocity measurements of $\mathbf{b}$ are performed. Scale bar, $1 \mathrm{~mm}$. b, Close-up on the head of a colloidal swarm propagating past random obstacles (black dots). The arrows are located at the colloid positions and point along the orientation of their velocity. Obstacle packing fraction:

$\phi_{0}=2.45 \times 10^{-2}$. Scale bar, $100 \mu \mathrm{m}$. c, Flocking phase diagram in the $\left(\rho, \phi_{0}\right)$ plane. The symbols represent the variations of $\phi_{0}^{\star}$ with $\rho$. Error bars: smaller than the symbols (defined as the difference between the minimal value of $\rho$ above which flocking was observed and the maximal value below which isotropic motion only was observed). $\mathbf{d}$, The $x$-component of the roller current is normalized by $J_{0}$ measured in an obstacle-free channel. $J_{x} / J_{0}$ is plotted as a function of the fraction of obstacles. Orientational order is suppressed in the shaded region. Error bars, 1 standard deviation (s.d.) (17 different flocks).

$J(r, t)$ projected on the $x$-axis, and averaged over time and space. Accordingly $J_{x}$ monotonically decreases with $\phi_{0}$ and vanishes at $\phi_{\mathrm{o}}^{\star}$ (Fig. 1d).

Our first goal is to elucidate this loss of orientational order. To do so, we consider the evolution of the flock morphology along the propagation direction upon increasing disorder. This morphology can be equivalently captured by the variations of the local density, current, or polarization fields, as demonstrated in the Supplementary Information. For sake of clarity we focus here 

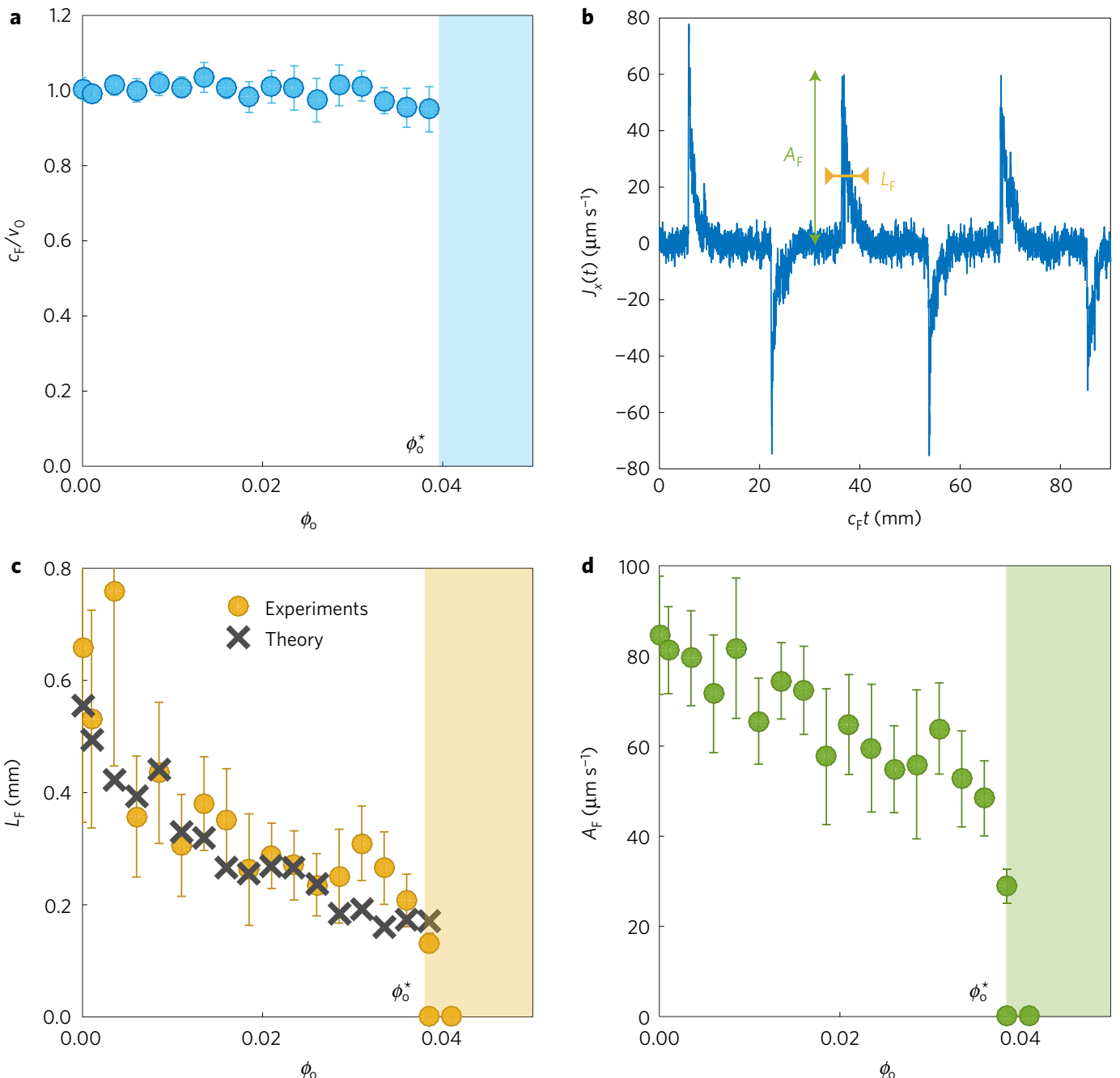

Figure 2 | Flock morphology. a, Flock speed normalized by the roller speed plotted as a function of the obstacle fraction. Error bars, 1 s.d. (17 flocks per data point). $\mathbf{b}$, Spatial variations of the longitudinal current. The shape of the steadily propagating flock is readily inferred from the temporal variations of the current averaged in a $25 \mu \mathrm{m} \times 1 \mathrm{~mm}$ rectangular region. The flock width $L_{F}$ is defined as the width of $J(t)$ at two-thirds of the maximal amplitude $A_{F}$. c, Yellow symbols: flock length plotted versus the obstacle fraction (averaged over 17 different flocks). Error bars, 1 s.d. Black symbols: analytical prediction of the flock length (see Supplementary Information). Shaded region: isotropic phase. d, Maximal amplitude of the longitudinal current plotted versus the obstacle fraction (averaged over 17 different flocks). Shaded region: isotropic phase. Note that at $\phi_{0}^{\star}$ both a flock state and a homogeneous isotropic state coexist. Error bars, 1 s.d.

on the roller current as the main observable. The flock speed $c_{\mathrm{F}}$ is unaltered by disorder and remains very close to the roller velocity for all $\phi_{\mathrm{o}}<\phi_{\mathrm{o}}^{\star}$ (Fig. 2a). Therefore, the time variations of $J_{x}(t) \equiv\langle\hat{\boldsymbol{x}} \cdot \boldsymbol{J}(x=0, y, t)\rangle_{y}$, the longitudinal current averaged over the transverse direction, give an accurate description of the coarsegrained shape of the flocks (Fig. 2b). Three important results are in order: the decrease of the flock length, $L_{\mathrm{F}}$, echoes that of the global order parameter and vanishes rather smoothly at $\phi_{\mathrm{o}}^{\star}$ (Fig. 2c). However, as shown in Fig. 2d, the maximal current amplitude, $A_{\mathrm{F}}$, undergoes a sharp drop and cancels discontinuously at $\phi_{0}^{\star}$. Finally, at $\phi_{0}^{\star}$, the flocks are intermittent: they repeatedly form and propagate steadily, before spontaneously vanishing and nucleating again. A featureless isotropic state coexists in time with a phaseseparated flocking state where macroscopic excitations as large as $1 \mathrm{~mm}$ propagate in the channel. Altogether these three observations yield firm evidence that disorder suppresses the flocking state in the form of a first-order non-equilibrium transition.

However, the obstacles do not merely reduce the extent of the flocks down to their extinction, but also trigger qualitative changes in their inner structure. The snapshots of the roller current $\boldsymbol{J}(\boldsymbol{r}, t)$ at four subsequent times in Fig. 3a demonstrate that the flocks are strongly heterogeneous spatial patterns (see also Supplementary Movie 4). We characterize the local flock morphology by introducing the current field $\boldsymbol{J}_{\text {flock }}(\boldsymbol{r})=\langle\boldsymbol{J}(\boldsymbol{r}, t)\rangle_{t \in \Delta t_{\mathrm{F}}}$ averaged over the time interval $\Delta t_{\mathrm{F}}$ taken by the flock to cross the observation window. At low $\phi_{0}$, we observe that colloiddepleted wakes as large as $\sim 50 \mu \mathrm{m}$ form downstream of each obstacle (see Fig. 3b, upper panel). However, as $\phi_{\mathrm{o}}$ increases, the competition between alignment interactions and multiple-obstacle scattering causes the redistribution of the roller current into a static river network (Fig. 3b). Virtually no collective motion occurs in the closed regions surrounded by the flowing rivers (black regions in Fig. 3b). The extent of the regions where the flow is suppressed can significantly exceed both the typical inter-obstacle distance and the depletion-wake size. Importantly, upon increasing $\phi_{0}$, the river network becomes increasingly sparse and different from the region of space left around the mere superposition of uncorrelated wakes. Comparing the areas of these two very different geometries allows us to quantify the sparsity of the river networks in Fig. 3c. In addition, the networks also become 

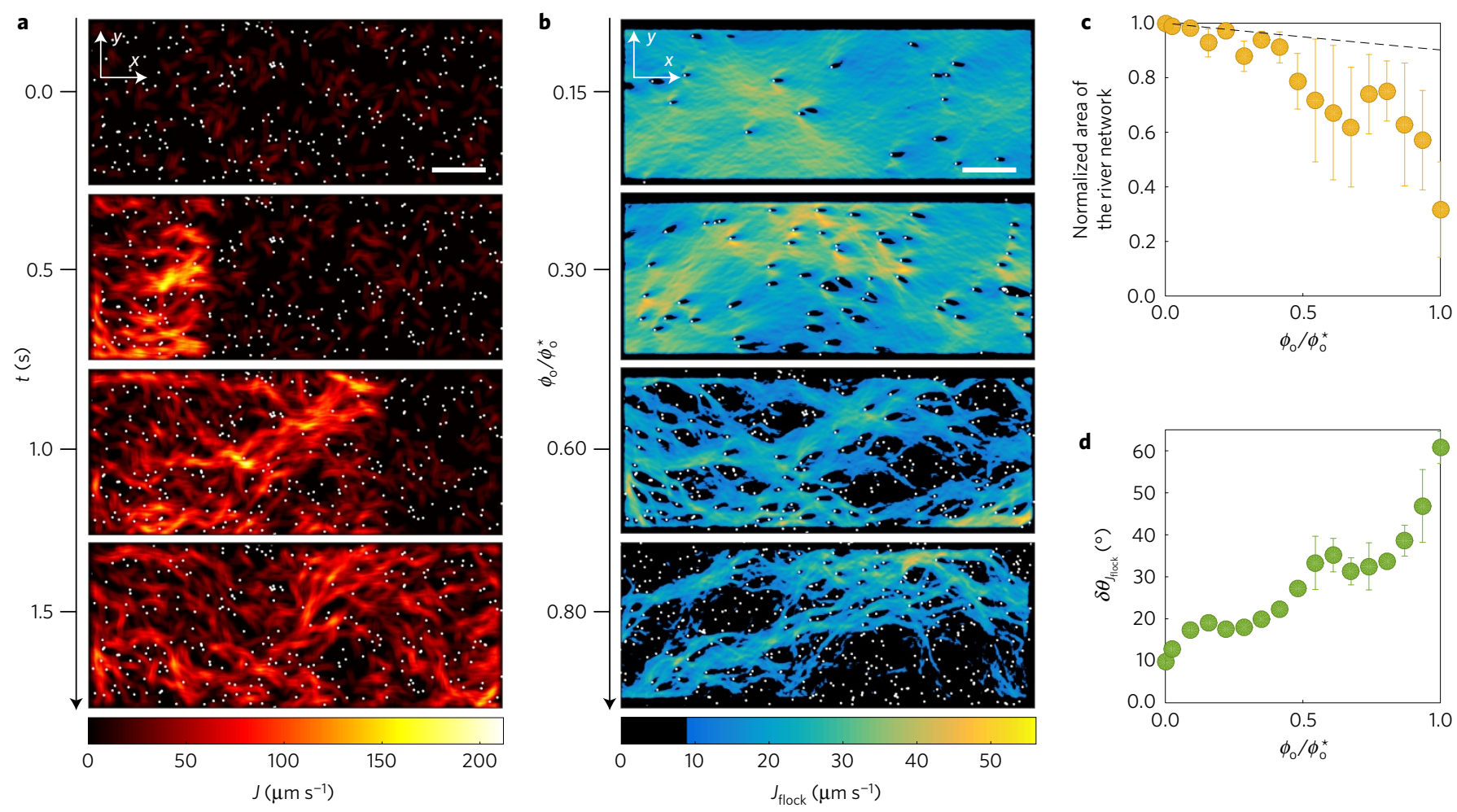

Figure 3 | Flocking through river networks. a, Four subsequent snapshots of the magnitude $|J(x, y, t)|$ of the current field. Coarse graining over $25 \mu \mathrm{m} \times 25 \mu \mathrm{m}$ bins. Time average over $0.05 \mathrm{~s} . \phi_{0} / \phi_{0}^{\star}=0.5$. The rollers flow along preferred channels. Scale bar, $200 \mu \mathrm{m}$. b, Magnitude of the flock current $J_{\text {flock }}$ plotted for four different obstacle packing fractions. At low $\phi_{0}$ colloid-depleted wakes form downstream of the obstacles. As $\phi_{0}$ increases, a channel network forms and becomes increasingly sparse and tortuous. Coarse graining over $12.5 \mu \mathrm{m} \times 12.5 \mu \mathrm{m}$ bins. Scale bar, $200 \mu \mathrm{m}$. c, Circles: Area of the flowing region normalized by the area of the observation window. Error bars, 1 s.d. (17 flocks per data point). Dashed line: area fraction of the region left around the superposition of spatially uncorrelated wakes. This quantity is computed knowing the fraction of space occupied by a random ensemble of patches having the shape of the wake formed downstream an isolated obstacle: $1-\exp \left(-\rho_{0} a_{\text {wake }}\right)$, where $\rho_{0}$ is the obstacle density and $a_{\text {wake }}$ the wake area ${ }^{36}$. d, The orientational fluctuations of $\boldsymbol{J}_{\text {flock }}$ sharply increase at the onset of flock destruction. They are defined as $\delta \theta_{J_{\text {flock }}}^{2}=A^{-1} \int \theta_{\text {flock }^{2}}^{2}(\boldsymbol{r}) \mathrm{d} \boldsymbol{r}$, where $A$ is the area of the observation region, and $\boldsymbol{J}_{\text {flock }} / J_{\text {flock }} \equiv\left(\cos \theta_{\mathrm{J}_{\text {flock }}}, \sin \theta_{\mathrm{fflock}_{\text {fock }}}\right)$. Error bars, 1 s.d. $(17$ flocks per data point $)$.

increasingly tortuous, as demonstrated by the rapid increase of the orientational fluctuations of $\boldsymbol{J}_{\text {flock }}$ with $\phi_{\mathrm{o}}$ in Fig. $3 \mathrm{~d}$. Above $\phi_{\mathrm{o}}^{*}$, orientational order survives to disorder in finite and short-lived rivers. Any form of macroscopic transport is suppressed, as these transient channels are isotropically distributed and do not percolate through the entire system (see Supplementary Movie 3 and the Supplementary Information). We close this discussion by stressing that these emergent river networks are strikingly similar to those encountered above the plastic depinning threshold when driving an ensemble of elastically coupled particles through quenched disorder (from vortex lattices in type-II superconductors, to driven colloids and grains $)^{21,24-31}$.

To elucidate the physics underlying the suppression of collective motion and the emergence of channelling networks, we first need a quantitative description of the roller-obstacle interactions. As a roller approaches an obstacle its direction of motion is repelled at a distance, yet its speed remains unchanged (Fig. 4a,b). The rollerobstacle and roller-roller repulsions stem from the same physical mechanisms ${ }^{10}$ : a dielectric obstacle causes a local radial perturbation of the electric field $\boldsymbol{E}=E_{0} \hat{z}$ used to power the Quincke rotation. As a result, a short-range repulsive torque reorients the roller velocity in the direction opposite to the obstacle (see Supplementary Information). This interaction has the same symmetry as that numerically considered in refs 15,32 . The scattering plots, shown in Fig. 4c and Supplementary Information, demonstrate that the repulsive torques are weak and short ranged. A head-on collision merely deflects the initial roller orientation by an angle of $60^{\circ}$.
As a consequence, up to $\phi_{\mathrm{o}}=0.1$, the trajectories in the isotropic phase remain diffusive at long times, and are fully characterized by their rotational diffusivity $D$, which increases linearly with $\phi_{\mathrm{o}}$ (Fig. 4d).

We can now account for the first-order nature of the flocking transition. The linear increase of $D$ suggests simplifying the interactions between the rollers and the obstacles as uncorrelated binary collisions with random scatterers ${ }^{32}$. Within this Boltzmann approximation, we can generalize the kinetic theory valid at the onset of collective motion, which we introduced in ref. 10. We show in the Supplementary Information that the roller-obstacle and roller-roller interactions decouple. Increasing the obstacle fraction solely renormalizes the angular noise acting on the rollers, even when they interact in the flock phase. We then readily conclude that the transition to collective motion should belong to the very same universality class as the first-order flocking transition found in all motile-spin models, starting from the seminal Vicsek model $^{33-35}$. We quantitatively test the relevance of this scenario by comparing, in Fig. 2c, the measured flock length to our theoretical prediction for the shape of such nonlinear excitations as detailed in the Supplementary Information. The unambiguous agreement confirms our theoretical explanation: weak quenched disorder triggers a generic Vicsek-like discontinuous transition from collective to isotropic motion.

However, this appealing scenario cannot capture the emergence of channelling networks at high obstacle fractions. We now theoretically account for these spatial fluctuations by describing the 
a

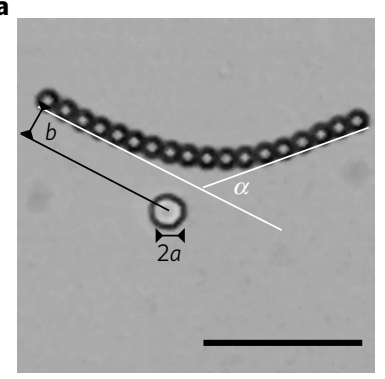

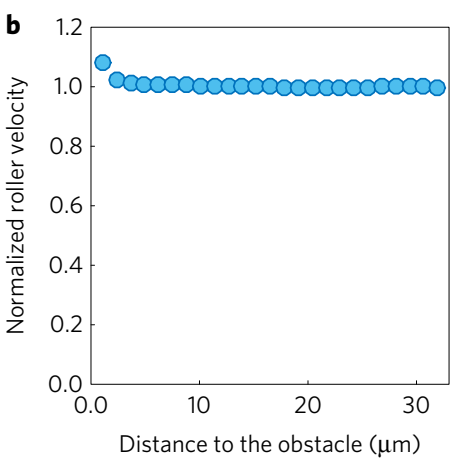

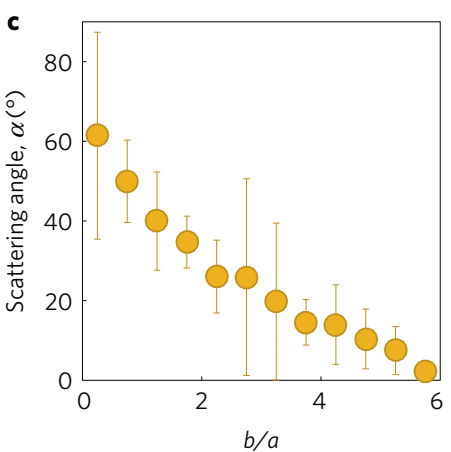

Figure 4 | Roller-obstacle scattering. a, Superimposed picture of a roller colliding with an obstacle of radius $a=5 \mu m$. $b$ is the impact parameter, $\alpha$ is the

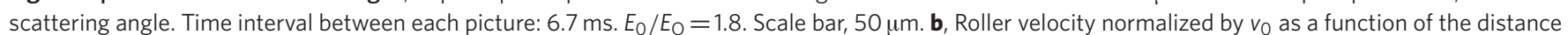

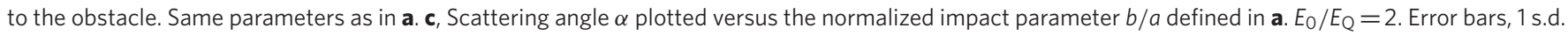
d, Rotational diffusivity defined as the decorrelation time of the roller velocity plotted as a function of the obstacle packing fraction (see Methods). $E_{0} / E_{Q}=2$. Error bars, 1 s.d.

strongly polarized region close to the flock front in the high- $\phi_{\mathrm{o}}$ regime. Therefore, rather than describing the obstacles as point-wise scatterers, we here consider small spatial fluctuations around a homogeneous obstacle-density field. The resulting hydrodynamic equations, derived in the Supplementary Information, are analogous to the Navier-Stokes equations for a polar active fluid:

$$
\begin{gathered}
\partial_{t} \rho+\nabla \cdot(\rho \boldsymbol{\Pi})=0 \\
\partial_{t} \boldsymbol{\Pi}+v_{0} \boldsymbol{\Pi} \cdot \nabla \boldsymbol{\Pi}=\mathbb{P} \cdot\left[-\beta \nabla \rho+\alpha_{2} \Delta(\rho \boldsymbol{\Pi})\right. \\
\left.+\gamma \tilde{\Delta} \cdot(\rho \boldsymbol{\Pi})+\boldsymbol{F}_{\mathrm{o}}\right]
\end{gathered}
$$

where we introduce the local polarization $\boldsymbol{\Pi}(\boldsymbol{r}, t) \equiv \boldsymbol{J}(\boldsymbol{r}, t) /$ $\left[v_{0} \rho(\boldsymbol{r}, t)\right]$, and $\mathbb{P}=\mathbb{I}-\boldsymbol{\Pi} \boldsymbol{\Pi}$. The convective term on the lefthand side of equation (2) stems from self-propulsion, $\beta \nabla \rho$ is a pressure term due to the repulsive interactions between the rollers, and $\alpha_{2}$ and $\gamma$ are the elastic constants of this polar liquid ( $\tilde{\Delta}$ is an anisotropic second-order operator). Finally, disorder is captured by the quenched force field $\boldsymbol{F}_{\mathrm{o}}(\boldsymbol{r})=-\beta_{0} \nabla \phi_{\mathrm{o}}(\boldsymbol{r})$ which localizes the rollers in the valleys of an effective potential given by the local obstacle-density field $\phi_{\mathrm{o}}(\boldsymbol{r})$. The linear response of $\boldsymbol{\Pi}=\hat{\boldsymbol{x}}+\delta \theta \hat{\boldsymbol{y}}$ provides a physical insight into the formation of sparse flowing channels. Within this approximation, the orientational fluctuations are readily computed from equations (1) and (2):

$$
\overline{\left|\delta \theta_{q}\right|^{2}}=\frac{\beta_{\mathrm{o}}^{2} \phi_{\mathrm{o}}}{v_{\mathrm{o}}^{2}+q_{x}^{2}\left(\alpha_{2}-\gamma\right)^{2} \rho^{2}}\left(\frac{q_{y}}{q_{x}}\right)^{2}
$$

where - stands for average over disorder, and $\boldsymbol{q}$ is a quasilongitudinal wave vector (see Supplementary Information). Equation (3) establishes that the polarization fluctuations are set by the competition between random stirring, self-propulsion and bending elasticity. Importantly, orientational fluctuations increase at all scales with the number of obstacles. However, the bending stiffness $\left(\alpha_{2}-\gamma\right)$ suppresses the small wavelength fluctuations required to explore the valleys of $\phi_{0}(r)$, which become increasingly branched and curved as the number of obstacles increases. This competition therefore selects a small subset of all the possible paths and consistently accounts for the formation of sparse and tortuous river networks upon increasing disorder. This scenario is further confirmed by experiments performed in periodic lattices of obstacles. By construction, these arrangements display minute density fluctuations. Therefore, the random stirring force in equation (2) is expected to be vanishingly small compared to an equally dense disordered medium. In agreement with our prediction, we find that no river network emerges in periodic lattices (see Supplementary Fig. 7 and Supplementary Movie 5). This scenario is expected to hold qualitatively beyond linear response. In addition, as it does not depend on the specifics of the colloidal rollers, it must be relevant to any flock made of motile bodies obstructed by repelling obstacles, from living-creature groups to swarming robots to soft-active materials.

\section{Methods}

Methods, including statements of data availability and any associated accession codes and references, are available in the online version of this paper.

Received 11 February 2016; accepted 31 August 2016; published online 3 October 2016

\section{References}

1. Kernbach, S. Handbook of Collective Robotics - Fundamentals and Challenges (Pan Stanford Edition, 2013).

2. Werfel, J., Petersen, K. \& Nagpal, R. Designing collective behavior in a termite-inspired robot construction team. Science 343, 754-758 (2014).

3. Berman, S., Radhika, N. \& Halasz, A. Optimization of stochastic strategies for spatially inhomogeneous robot swarms: a case study in commercial pollination. Proceedings of IEEE/RSI International Conference on Intelligent Robots and Systems (IROS’11)3923-3930 (IEEE Computer Society Press, 2011).

4. Shklarsh, A., Ariel, G., Schneidman, E. \& Ben-Jacob, E. Smart swarms of bacteria-inspired agents with performance adaptable interactions. PLoS Comput. Biol. 7, e1002177 (2011).

5. Schaller, V., Weber, C., Semmrich, C., Frey, E. \& Bausch, A. Polar patterns of driven filaments. Nature 467, 73-77 (2010).

6. Deseigne, J., Dauchot, O. \& Chaté, H. Collective motion of vibrated polar disks. Phys. Rev. Lett. 105, 098001 (2010).

7. Thutupalli, S., Seemann, R. \& Herminghaus, S. Swarming behavior of simple model squirmers. New J. Phys. 13, 073021 (2011).

8. Sanchez, T., Chen, D. T. N., DeCamp, S., Heymann, M. \& Dogic, Z. Spontaneous motion in hierarchically assembled active matter. Nature 491, 431-435 (2012).

9. Theurkauff, I., Cottin-Bizonne, C., Palacci, J., Ybert, C. \& Bocquet, L. Dynamic clustering in active colloidal suspensions with chemical signaling. Phys. Rev. Lett. 108, 268303 (2012).

10. Bricard, A., Caussin, J.-B., Desreumaux, N., Dauchot, O. \& Bartolo, D. Emergence of macroscopic directed motion in populations of motile colloids. Nature 503, 95-98 (2013).

11. Palacci, J., Sacanna, S., Steinberg, A. P., Pine, D. J. \& Chaikin, P. M. Living crystals of light-activated colloidal surfers. Science 339, 936-940 (2013).

12. Buttinoni, I. et al. Dynamical clustering and phase separation in suspensions of self-propelled colloidal particles. Phys. Rev. Lett. 23, 238301 (2013).

13. Lushi, E., Wioland, H. \& Goldstein, R. E. Fluid flows created by swimming bacteria drive self-organization in confined suspensions. Proc. Natl Acad. Sci. USA 111, 9733-9738 (2014). 
14. Nishiguchi, D. \& Sano, M. Mesoscopic turbulence and local order in Janus particles self-propelling under an ac electric field. Phys. Rev. E 92, 052309 (2015).

15. Chepizhko, O., Altmann, E. G. \& Peruani, F. Optimal noise maximizes collective motion in heterogeneous media. Phys. Rev. Lett. 110, 238101 (2013).

16. Reichhardt, C. \& Olson Reichhardt, C. J. Active matter transport and jamming on disordered landscapes. Phys. Rev. E 90, 012701 (2014).

17. Quint, D. A. \& Gopinathan, A. Topologically induced swarming phase transition on a 2D percolated lattice. Phys. Biol. 12, 046008 (2015).

18. Marchetti, M. C. et al. Hydrodynamics of soft active matter. Rev. Mod. Phys. 85, 1143-1189 (2013).

19. Vicsek, T. \& Zafeiris, A. Collective motion. Phys. Rep. 517, 71-140 (2012).

20. Cavagna, A. \& Giardina, I. Bird flocks as condensed matter. Annu. Rev. Condens. Matter Phys. 5, 183-207 (2014).

21. Reichhardt, C. \& Olson, C. J. Depinning and nonequilibrium dynamic phases of particle assemblies driven over random and ordered substrates: a review. Preprint at http://arxiv.org/abs/1602.03798v1 (2016).

22. Bricard, A. et al. Emergent vortices in populations of colloidal rollers. Nat. Commun. 6, 7470 (2015).

23. Quincke, G. Über Rotationen im constanten electrischen Felde. Ann. Phys. 295, 417-486 (1896)

24. Jensen, H. J., Brass, A. \& Berlinsky, A. J. Lattice deformations and plastic flow through bottlenecks in a two-dimensional model for flux pinning in type-II superconductors. Phys. Rev. Lett. 60, 1676-1679 (1988).

25. Grønbech-Jensen, N., Bishop, A. R. \& Domínguez, D. Metastable filamentary vortex flow in thin film superconductors. Phys. Rev. Lett. 76, 2985-2988 (1996).

26. Troyanovski, A. M., Aarts, J. \& Kes, P. H. Collective and plastic vortex motion in superconductors at high flux densities. Nature 399, 665-668 (1999).

27. Le Doussal, P. \& Giamarchi, T. Moving glass theory of driven lattices with disorder. Phys. Rev. B 57, 11356-11403 (1998).

28. Faleski, M. C., Marchetti, M. C. \& Middleton, A. A. Vortex dynamics and defects in simulated flux flow. Phys. Rev. B 54, 12427-12436 (1996).

29. Topinka, M. A. et al. Coherent branched flow in a two-dimensional electron gas. Nature 410, 183-186 (2001).
30. Pertsinidis, A. \& Ling, X. S. Statics and dynamics of 2D colloidal crystals in a random pinning potential. Phys. Rev. Lett. 100, 028303 (2008).

31. Yan, L., Barizien, A. \& Wyart, M. A model for the erosion onset of a granular bed sheared by a viscous fluid. Phys. Rev. E 93, 012903 (2015).

32. Chepizhko, O. \& Peruani, F. Diffusion, subdiffusion, and trapping of active particles in heterogeneous media. Phys. Rev. Lett. 111, 160604 (2013).

33. Solon, A. P., Chaté, H. \& Tailleur, J. From phase to microphase separation in flocking models: the essential role of nonequilibrium fluctuations. Phys. Rev. Lett. 114, 068101 (2015).

34. Vicsek, T., Czirók, A., Ben-Jacob, E., Cohen, I. \& Shochet, O. Novel type of phase transition in a system of self-driven particles. Phys. Rev. Lett. 75, 1226-1229 (1995).

35. Grégoire, G. \& Chaté, H. Onset of collective and cohesive motion. Phys. Rev. Lett. 92, 025702 (2013).

36. Kellerer, A. M. On the number of clumps resulting from the overlap of randomly placed figures in a plane. J. Appl. Probab. 20, 126-135 (1983).

\section{Acknowledgements}

We acknowledge support from ANR program MiTra and Institut Universitaire de France. We thank F. Peruani, S. Santucci, M. C. Marchetti and D. Carpentier for valuable comments and suggestions. We also thank G. Fabre for help with the experiments.

\section{Author contributions}

D.B. conceived the project. N.D., A.M. and D.B. designed the experiments. N.D. and A.M. performed the experiments. J.-B.C. and D.B. performed the theory. N.D., A.M., J.-B.C. and D.B. analysed and discussed results. D.B. and A.M. wrote the paper. N.D. and A.M. have equal contributions.

\section{Additional information}

Supplementary information is available in the online version of the paper. Reprints and permissions information is available online at www.nature.com/reprints.

Correspondence and requests for materials should be addressed to D.B.

\section{Competing financial interests}

The authors declare no competing financial interests. 


\section{Methods}

We use fluorescent polystyrene colloids of diameter $4.8 \mu \mathrm{m}$ dispersed in a $0.15 \mathrm{moll}^{-1}$ AOT-hexadecane solution (Thermo Scientific G0500). The suspension is injected in a wide microfluidic chamber made of two parallel glass slides coated by a conducting layer of indium tin oxide (ITO) (Solems, ITOSOL30, thickness: $80 \mathrm{~nm})^{10}$. The two electrodes are assembled with double-sided Scotch tape of homogeneous thickness $(110 \mu \mathrm{m})$. The colloids are confined in a $1 \mathrm{~mm} \times 15 \mathrm{~mm}$ channel, by walls made of a positive photoresist resin (Microposit S1818, thickness: $2 \mu \mathrm{m}$ ). Identical cylindrical obstacles of radius $5 \mu \mathrm{m}$ made of the same material are included in the main channel. Their position is uniformly distributed with a density $\rho_{0}$, and the obstacle fraction is defined as $\phi_{0}=\pi a^{2} \rho_{\mathrm{o}}$ in the main text. Note that some of the obstacles overlap. This geometry is achieved by means of conventional ultraviolet lithography. More details about the design of the microfluidic device are provided in the Supplementary Methods.

The Quincke electro-rotation of the colloids is controlled by applying a homogeneous electric field transverse to the two electrodes $\boldsymbol{E}=E_{0} \hat{\boldsymbol{z}}$. The field is applied with a voltage amplifier (TREK 609E-6). All the reported results correspond to an electric field $E_{0}=2 E_{\mathrm{O}}$, where $E_{\mathrm{O}}$ is the Quincke electro-rotation threshold $E_{\mathrm{Q}}=1 \mathrm{~V} \mu \mathrm{m}^{-1}$. All the measurements are performed when a steady state is reached for all the observables. The colloids are observed with a $7.2 \times$ magnification with a fluorescent Nikon AZ100 microscope. The movies are recorded with a CMOS camera (Basler ACE) at frame rates of $380 \mathrm{fps}$. The particles are detected to sub-pixel accuracy, and the particle trajectories and velocities are reconstructed using the Crocker and Grier algorithm ${ }^{37}$. Measurements are performed in a $1.22 \mathrm{~mm} \times 0.75 \mathrm{~mm}$ observation window. All measurements have been systematically repeated for 15 to 18 different flocks crossing the same field of view (different initial conditions). In addition we have used four different realizations of the disordered arrangements of obstacles. The observation window was set close to the midpoint of the main channel where all the morphological quantities have reached their stationary values. Measurements performed further away from the walls yield identical results.

All the colloids roll at constant speed $v_{0}=1.4 \pm 0.1 \mathrm{~mm} \mathrm{~s}^{-1}$. When isolated, their direction of motion freely diffuses on the unit circle with a diffusivity $D=1.6 \pm 0.1 \mathrm{~s}^{-1}$. $D$ is defined as the exponential decorrelation rate of the velocity orientation in a isotropic phase, $\left\langle\hat{\boldsymbol{v}}_{i}(0) \cdot \hat{\boldsymbol{v}}_{i}(t)\right\rangle_{i} \sim \exp (-D t)$, where $\hat{\boldsymbol{v}}_{i}$ is the velocity orientation of the ith roller.

The current field $J(r, t)$ is computed by summing the instantaneous roller velocities in $12.5 \mu \mathrm{m} \times 12.5 \mu \mathrm{m}$ binning windows. The flock current $\boldsymbol{J}_{\text {flock }}(\boldsymbol{r})$ is computed by averaging $\boldsymbol{J}(\boldsymbol{r}, t)$ over time. The flowing-path network is defined as the ensemble of points where $J_{\text {flock }}$ exceeds $11 \mu \mathrm{m} \mathrm{s}^{-1}$. This value has been chosen as the typical average current in the wake left behind an isolated obstacle. None of the results discussed in this letter qualitatively depend on this specific threshold value. The current-free regions referred to in the main text are associated with local current values smaller than this threshold (black areas in Fig. 3b).

Data availability. The data that support the plots within this paper and other findings of this study are available from the corresponding author upon request.

\section{References}

37. Crocker, J. C. \& Grier, G. Methods of digital video microscopy for colloidal studies. J. Colloid Interface Sci. 179, 298-310 (1996). 